\title{
Role of iron, zinc and reduced glutathione in oxidative stress induction by low $\mathrm{pH}$ in rat brain synaptosomes
}

\author{
Tatyana G Pekun', Sviatlana V Hrynevich', Tatyana V Waseem² and Sergei V Fedorovich ${ }^{\text {** }}$
}

\begin{abstract}
Brain ischemia leads to a decrease in $\mathrm{pH}_{\mathrm{o}}$. We have shown previously in synaptosomes that the extracellular acidification induces depolarization of mitochondria followed by synthesis of superoxide anions and oxidative stress. Here, we investigated the effects of lowered $\mathrm{pH}_{\circ}$ on oxidative stress and membrane potentials in synaptosomes treated by the iron chelator deferoxamine and zinc chelator TPEN. We demonstrated that chelating of metals has no impact on superoxide anion synthesis and intrasynaptosomal mitochondria depolarization. Meanwhile, deferoxamine was able to inhibit oxidative stress induced by low $\mathrm{pH}_{0}$ and hydrogen peroxide application. Compared to deferoxamine, TPEN was less effective but it decreased the DCF fluorescence induced by $\mathrm{pH}_{\mathrm{o}} 6.0$ which had no effects in other oxidative stress models. We found that the chelators were able to inhibit slightly plasma membrane depolarization. Synaptosomes preincubation at low $\mathrm{pH}_{\mathrm{o}}$ caused no effects on the reduced glutathione level. Depletion of glutathione by CDNB produced no additional increase in the DCF fluorescence induced by $\mathrm{pH}_{\circ}$ 7.0. Our results suggest that free iron is crucial for the development of oxidative stress elicited by acidification in synaptosomes. Chelating of this metal seems to be a promising strategy for protecting the neuronal presynaptic terminals against oxidative stress developed at stroke.
\end{abstract}

Keywords: Synaptosomes; Acidosis, ischemia; Iron; Zinc; Glutathione

\section{Introduction}

Stroke is associated with acidification reaching $\mathrm{pH}_{\mathrm{o}}$ of 5.3 in certain cases, for instance, in hyperglycemia (Thorn and Heitmann 1954; Crowell and Kaufman, 1961; Kraig and Chesler 1990; Tombaugh and Sapolsky 1993; Isaev et al. 2008). The main cause of $\mathrm{pH}$ lowering is a metabolic shift to predominance of glycolysis (Tombaugh and Sapolsky 1993; Isaev et al. 2008; Obara et al. 2008). Apart form ischemia, acidification was also observed in several neurodegenerative diseases (Yates et al. 1990) potentially contributing to their pathogenesis.

Lowering of $\mathrm{pH}$ down to 6.0 can induce neuronal death (Nedergaard et al. 1991; Isaev et al. 2010). The main cause of acid-induced neuronal death is thought to be activation of the acid sensitive ion channels (ASICs) (Krishtal and Pidoplichko 1981; Xiong et al. 2004; Isaev et al. 2008;

\footnotetext{
* Correspondence: fedorovich@ibp.org.by

${ }^{1}$ Laboratory of Biophysics and Engineering of Cell, Institute of Biophysics and Cell Engineering, Akademicheskaya St., 27, Minsk 220072, Belarus Full list of author information is available at the end of the article
}

Wemmie et al. 2013). However, at least in some cases, damage of neurons under low $\mathrm{pH}$ was associated rather with an acidification-induced increase in cytosolic zinc levels than ASIC activity (Isaev et al. 2010; Kiedrowski 2011). It was suggested that the mitochondria depolarization followed by oxidative stress plays a key role in development of this phenomenon (Isaev et al. 2010).

It was shown that lowering of $\mathrm{pH}$ may lead to an increase of free radical formation in the brain homogenates, slices and isolated neuronal presynaptic terminals termed synaptosomes (Siesjo et al. 1985; Bralet et al. 1991, 1992; Pekun et al. 2012, 2013).

Recently, we have demonstrated that superoxide anion synthesis in mitochondria followed by their depolarization is the primary cause of oxidative stress induced by extracellular acidification (Pekun et al. 2013). Nonetheless, the release of iron from proteins and inhibition of enzymes maintaining the cellular pool of reduced glutathione were reported to exert the crucial effects on development of oxidative stress in brain homogenates, brain slices, and 
neurons (Siesjo et al. 1985; Bralet et al. 1992; Ying et al. 1999; Lewerenz et al. 2010). It is unknown whether iron, zinc and glutathione have any role in the development of oxidative stress in synaptosomes, an experimental model that we have characterized earlier (Pekun et al. 2012, 2013). Meanwhile, it was demonstrated that the local free radical formation in synapses is able to modify significantly the synaptic vesicle recycling (Giniatullin et al. 2006; Keating 2008; Tarasenko et al. 2012; Tsentsevitsky et al. 2013). Accordingly, synaptic oxidative stress induced by low $\mathrm{pH}_{\mathrm{o}}$ might underlay irreversible impairment of synaptic transmission which is a poorly investigated consequence of brain ischemia (Hofmeijer and van Putten 2012).

In the present, paper we investigated an impact of the membrane permeable iron chelator deferoxamine and membrane permeable zinc chelator N,N,N,N'-tetrakis(2pyridylmethyl)ethylenediamine (TPEN) on free radical formation in rat brain synaptosomes at low $\mathrm{pH}_{\mathrm{o}}$. Reactive oxygen species (ROS) accumulation was monitored by the fluorescent dye DCFDA and dihydroethidium (LeBel and Bondy 1990; Pekun et al. 2013). It is well known that depolarization of mitochondrial membrane can cause free radical formation (Votyakova and Reynolds 2001; Abramov et al. 2007; Manzanero et al. 2013; Pekun et al. 2013) and a subsequent ROS accumulation is able to result in depolarization of the neuronal plasma membrane (Bao et al. 2005; Nani et al. 2010). Acidification decreases potentials in either mitochondrial or plasma membrane of rat brain synaptosomes (Fedorovich et al. 1996, 2003; Pekun et al. 2013, 2014); therefore, we investigated the effects of chelators on mitochondrial or plasma membrane potentials. Plasma membrane potential was monitored by a fluorescent dye DiSC3(5) (Waseem and Fedorovich 2010), mitochondrial potential was monitored by a fluorescent dye JC-1 (Chinopoulos et al. 1999). Further, we investigated the intrasynaptosomal concentration of reduced glutathione after lowering of pHo. Glutathione was monitored by a fluorescent dye monochlorobimane (Kamencic et al. 2000; Abramov et al. 2007).

\section{Materials and methods \\ Materials}

Dihydroethidium, 2,7'-dichlorodihydrofluorescein diacetate (DCFDA), oligomycin, 3,3' - dipropylthiadicarbocyanine (DiSC3(5)), deferoxamine mesylate, butylated hydroxytoluene (ionol), 1-chloro-2,4-dinitrobenzene (CDNB), monochlorobimane and N, N, N', N'-tetrakis(2-pyridylmethyl) ethylenediamine (TPEN) were purchased from Sigma (St. Louis, MO, USA). 4-(2-Hydroxyethyl)piperazine-N'1-ethanesulfonic acid (HEPES) was obtained from Merck (Darmstadt, Germany). 5,5'6,6'-tetrachloro-1,1,3,3'-tetraethylbenzimidazolo-carbocyanine iodide (JC-1) and rotenone were received from Calbiochem (La Jolla, CA,
USA). 4-morpholineethanesulfonic acid (MES) was purchased from Reanal (Budapest, Hungary). Tris(hydroxymethyl)aminomethane (Tris) was obtained from $\mathrm{BDH}$ (Poole, UK).

\section{Synaptosomes preparation}

Synaptosomes were isolated from brain hemispheres of 12-16-week-old male Wistar rats according to Hajos (1975). Stock suspensions of synaptosomes $(10 \mathrm{mg} / \mathrm{ml})$ were prepared in medium A (composition in mM: 132 $\mathrm{NaCl}, 5 \mathrm{KCl}, 10$ glucose, $1.3 \mathrm{MgCl}_{2}, 1.2 \mathrm{NaH}_{2} \mathrm{PO}_{4}, 15$ HEPES, 5 Tris, pH 7.4, $310 \mathrm{mOsm} / \mathrm{l}$ ) and kept on ice. Animal experiments were carried out in accordance with EU Directive 2010/63/EU.

\section{Intrasynaptosomal ROS determination}

Intrasynaptosomal ROS was monitored by fluorescent dye DCFDA according to LeBel and Bondy (1990) with modifications according to Alekseenko et al. (2008).

Synaptosomes purification was carried out in medium A and then after additional washing the pellet was resuspended in the same medium (protein concentration 10 $\mathrm{mg} / \mathrm{ml}$ ). Suspension was incubated for $60 \mathrm{~min}$ at $37^{\circ} \mathrm{C}$ in presence of $25 \mu \mathrm{M}$ DCFDA. Extracellular dye was removed by sedimentation and the final pellet was resuspended in 2 $\mathrm{ml}$ calcium-free medium B (composition in mM: $132 \mathrm{NaCl}$, $5 \mathrm{KCl}, 10$ glucose, $1.3 \mathrm{MgCl}_{2}, 1.2 \mathrm{NaH}_{2} \mathrm{PO}_{4}, 2.0 \mathrm{CaCl}_{2}, 10$ HEPES, $10 \mathrm{MES}$, pH 6.0-7.4, $310 \mathrm{mOsm} / \mathrm{l})$. To investigate ROS formation, $200 \mu \mathrm{l}$ of loaded synaptosomes were added to the cuvette containing $1.8 \mathrm{ml}$ of incubation medium $\mathrm{B}$ Fluorescence intensity was recorded at $\lambda_{\text {ex/em }}=501 / 525$ $\mathrm{nm}$ on spectrofluorimeter Cary Eclipse ("Varian", USA) with constant stirring and $37^{\circ} \mathrm{C}$ temperature.

To change the extracellular $\mathrm{pH}$, the aliquots of $60 \mu \mathrm{l}$ of $\mathrm{HCl}$ solution having different acid concentrations were directly added to the cuvette at $50 \mathrm{~s}$. The same quantity of water was added in control experiments. The control curve was extracted from the experimental curve.

\section{Determination of superoxide anion formation}

Superoxide anion level was determined by fluorescent dye dihydroethidium according to Pekun et al. (2013).

Synaptosomes purification was carried out in calciumfree medium A. Synaptosomal pellet was resuspended in calcium-free medium B. An aliquot of synaptosome suspension $(200 \mu \mathrm{l})$ was added to the cuvette containing 1.8 $\mathrm{ml}$ of incubation medium $\mathrm{B}$ with $2.0 \mathrm{mM} \mathrm{CaCl}_{2} .5 \mu \mathrm{M}$ of dihydroethidium were added to the cuvette, then after 1 minute different additions were made. Fluorescence intensity was recorded at $\lambda_{\text {ex/em }}=490 / 560 \mathrm{~nm}$ on spectrofluorimeter Cary Eclipse ("Varian", USA) at constant stirring and $37^{\circ} \mathrm{C}$.

To change the extracellular $\mathrm{pH}$ the aliquots of $60 \mu \mathrm{l}$ of $\mathrm{HCl}$ solution having different acid concentrations were 
added to the cuvette directly. The same quantity of water was added in control experiments. The control curve was extracted from the experimental curve.

\section{Determination of intrasynaptosomal mitochondria membrane potential by fluorescent dye JC-1}

Membrane potential of intrasynaptosomal mitochondria was detected by fluorescent dye JC-1 according to Chinopoulos et al. (1999) with modifications according to Pekun et al. (2013).

Synaptosomes purification was carried out in calciumfree medium $A$ and then the pellet was resuspended in the same medium (protein concentration of $5 \mathrm{mg} / \mathrm{ml}$ ). Suspension was incubated for $15 \mathrm{~min}$ at $37^{\circ} \mathrm{C}$ in the presence of $10 \mu \mathrm{g} / \mathrm{ml}$ dye. Extracellular dye was washed out three times by sedimentation and the final pellet was resuspended in $2.0 \mathrm{ml}$ calcium-free medium B (protein concentration of $10 \mathrm{mg} / \mathrm{ml}$ ).

To investigate mitochondrial membrane potential, 200 $\mu \mathrm{l}$ of loaded synaptosomes were added to the cuvette containing $1.8 \mathrm{ml}$ of incubation medium B. Fluorescence intensity was recorded at $\lambda_{\text {ex } / \mathrm{em}}=504 / 535 \mathrm{~nm}$ on spectrofluorimeter Cary Eclipse ("Varian", USA) at constant stirring and $37^{\circ} \mathrm{C}$.

To change the extracellular $\mathrm{pH}, 60 \mu \mathrm{l}$ of $\mathrm{HCl}$ solutions having different acid concentrations were directly added to the cuvette on 50s. The same quantity of water was added in control experiments. The control curve was extracted from the experimental curve.

\section{Investigation of plasma membrane potential}

Plasma membrane potential was investigated using fluorescent dye 3,3'-dipropylthiadicarbocianyne (DiSC3(5)) according to Waseem and Fedorovich (2010). An aliquot of synaptosome suspension $(200 \mu \mathrm{l})$ was added to the cuvette containing $2 \mathrm{ml}$ of incubation medium B. After $1 \mathrm{~min} 1$ $\mu \mathrm{M}$ of DiSC3(5) was added to the cuvette. After 1 minute, $10 \mu \mathrm{M}$ of rotenone and $5 \mu \mathrm{g} / \mathrm{ml}$ oligomycin was added. Fluorescence intensity was recorded at $\lambda_{\text {ex/em }}=640 / 688$ nm on spectrofluorimeter Cary Eclipse ("Varian", USA) at constant stirring and $37^{\circ} \mathrm{C}$. Synaptosomes were preincubated with different chelators for $30 \mathrm{~min}$ at $37^{\circ} \mathrm{C}$. All indicated compounds also were present in incubation medium throughout the fluorescence measurements.

To change the extracellular $\mathrm{pH}, 60 \mu \mathrm{l}$ of $\mathrm{HCl}$ solutions having different acid concentrations were directly added to the cuvette in 1 minute after addition of rotenone and oligomycin. The same quantity of water was added in control experiments. The control curve was extracted from the experimental curve.

\section{Determination of reduced glutathione}

Level of reduced glutathione was estimated by fluorescent dye monochlorobimane (Kamencic et al. 2000; Abramov et al. 2007). Synaptosomes purification was carried out in calcium-free medium A. Synaptosomal pellet was resuspended in calcium-free medium B. An aliquot of synaptosomal suspension $(200 \mu \mathrm{l})$ was added to $800 \mu \mathrm{l}$ incubation medium B having $\mathrm{pH} 7.4,7.0,6.0$ or containing $50 \mu \mathrm{M}$ of 1-chloro-2,4-dinitrobenzene (CDNB). Synaptosomes were sedimented by centrifugation after $10 \mathrm{~min}$ incubation at $37^{\circ} \mathrm{C}$. Then pellets were resuspended in $1 \mathrm{ml}$ of incubation medium A containing $50 \mu \mathrm{M}$ of monochlorobimane. Samples were incubated for $40 \mathrm{~min}$ at room temperature. The reaction was stopped by transferring samples on ice followed by fast centrifugation. The resulting pellets was resuspended again in $2 \mathrm{ml}$ of incubation medium $\mathrm{A}$, and fluorescence was measured on spectrofluorimeter Cary Eclipse ("Varian", USA) at $\lambda_{\mathrm{ex} / \mathrm{em}}=383 / 485 \mathrm{~nm}$.

\section{Other methods}

Protein concentration was assayed according to (Lowry et al. 1951) using bovine serum albumin as a standard. Data are presented as mean \pm S.E.M. where indicated, statistical significance was evaluated using one-tailed Student's t-test.

\section{Results}

Role of iron and zinc in development of oxidative stress

Figure 1a illustrates that decreasing of $\mathrm{pH}_{\mathrm{o}}$ down to 6.0 results in an increase in DCF fluorescence, as we had demonstrated earlier (Pekun et al. 2013). As expected, this $\mathrm{pH}_{\mathrm{o}}$-induced increase was sensitive to the antioxidant ionol $(200 \mu \mathrm{M})$ (Figure $1 \mathrm{~b})$. In these experimental conditions, oxidative stress was inhibited by the iron chelator deferoxamine $(100 \mu \mathrm{M})$ and zinc chelator TPEN (5 $\mu \mathrm{M})$, with deferoxamine being even more effective than ionol (Figure 1b). Conversely, oxidative stress induced by a moderate acidification $\left(\mathrm{pH}_{\mathrm{o}} 7.0\right)$ was sensitive to deferoxamine, but not to TPEN (Figure 1c). The increase in DCF fluorescence induced by $1 \mathrm{mM} \mathrm{H}_{2} \mathrm{O}_{2}$ was sensitive to ionol and deferoxamin, but not to TPEN (Figure 1d).

\section{Role of iron and zinc in superoxide anion synthesis}

Figure $2 \mathrm{a}$ shows that decreasing of $\mathrm{pH}_{\mathrm{o}}$ down to 6.0 results in the elevation dihydroethidium fluorescence, as we had demonstrated earlier (Pekun et al. 2013). This effect was not abolished by application of iron and zinc chelators (Figure 2b).

\section{Role of iron and zinc in induction of intrasynaptosomal mitochondria depolarization}

Figure 3a shows that decreasing of $\mathrm{pH}_{\mathrm{o}}$ down to 6.0 results in the elevation JC-1 fluorescence, as we had demonstrated earlier (Pekun et al. 2013). This effect was not abolished by application of iron and zinc chelators (Figure 3b). 

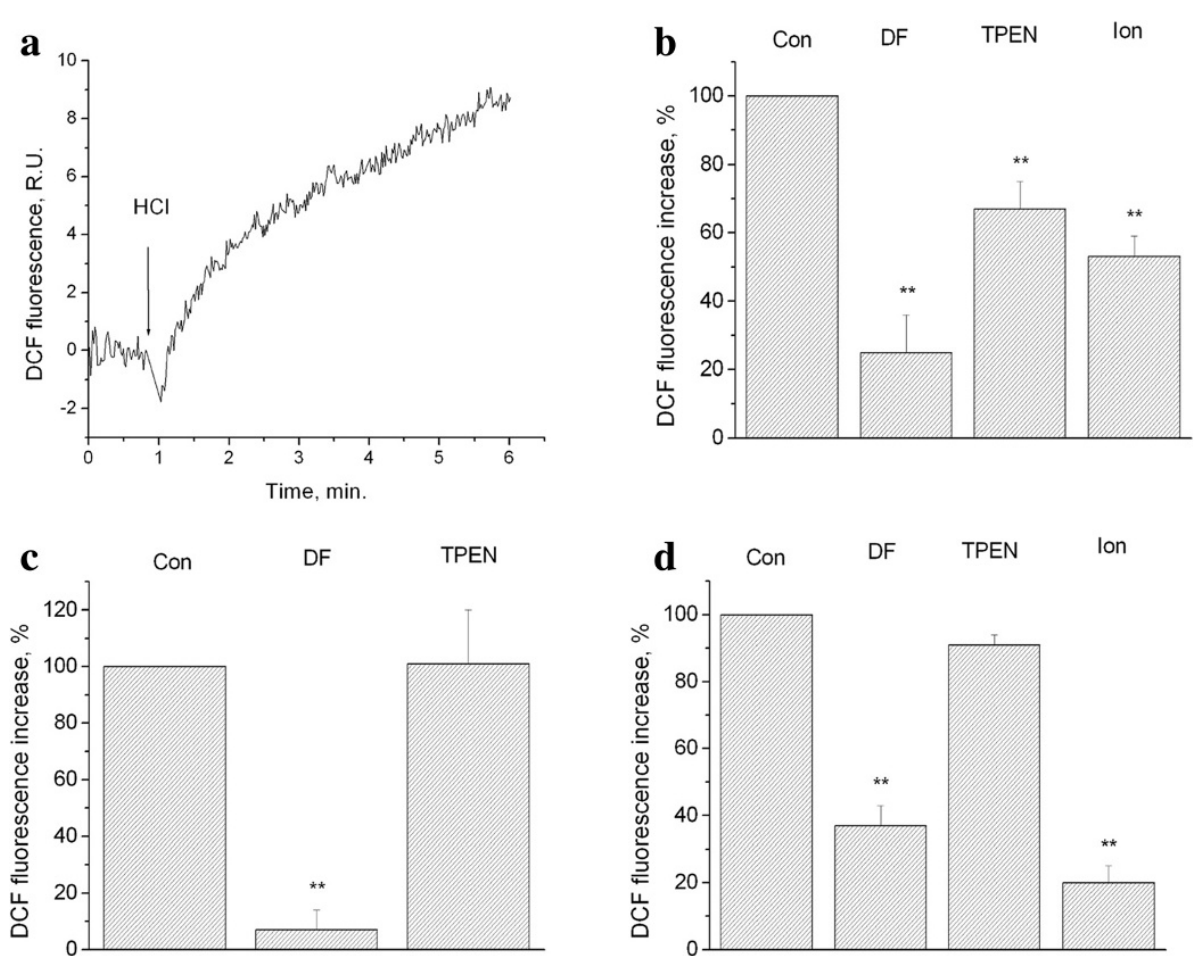

Figure 1 Influence of extracellular pH on DCF fluorescence in synaptosomes. Role of iron and zinc. a) Kinetics of DCF fluorescence increase after extracellular acidification. $\mathrm{HCl}$ down to $\mathrm{pH} 6.0$ was added where indicated. Curves represent 5 independent experiments. b) Influence of deferoxamine, TPEN and ionol on DCF fluorescence evoked by pH 6.0 c) Influence of desferoxamine and TPEN on DCF fluorescence evoked by $\mathrm{pH} 7.0$ d) Influence of desferoxamine, TPEN and ionol on DCF fluorescence evoked by $1 \mathrm{mM} \mathrm{H}_{2} \mathrm{O}_{2}$. Con - control DF - synaptosomes were preincubated for $60 \mathrm{~min}$ at $37^{\circ} \mathrm{C}$ with $100 \mu \mathrm{M}$ of deferoxamine, incubation medium contains also $100 \mu \mathrm{M}$ of desferoxamine. TPEN - synaptosomes were preincubated for $60 \mathrm{~min}$ at $37^{\circ} \mathrm{C}$ with $5 \mu \mathrm{M}$ of TPEN, incubation medium contains also $5 \mu \mathrm{M}$ of TPEN lon - synaptosomes were preincubated for $60 \mathrm{~min}$ at $37^{\circ} \mathrm{C}$ with $200 \mu \mathrm{M}$ of ionol, incubation medium contains also $200 \mu \mathrm{M}$ of ionol. Bars represent DCF fluorescence increase within 4 minutes after additions. Data presented are mean values \pm SEM of at least 4 experiments. $100 \%$ level corresponds to fluorescence increase in response to $\mathrm{pH} 6.0$ (b), pH 7.0 (c), 1 mM of $\mathrm{H}_{2} \mathrm{O}_{2}$ (d) without chelators and antioxidants (b), ${ }^{*} \mathrm{P} \leq 0.01$ vs. $100 \%$.

\section{Role of iron and zinc in induction of plasma membrane depolarization}

Figure 4a shows that decreasing of $\mathrm{pH}_{\mathrm{o}}$ down to 6.0 results in the elevation DiSC3(5) fluorescence, as we had demonstrated earlier (Pekun et al. 2014). This effect was sensitive to iron and zinc chelators (Figure 4b). Furthermore, TPEN was more effective than deferoxamine (Figure $4 \mathrm{~b}$ ).

\section{Role of reduced glutathione in induction of oxidative stress}

Figure 5 shows that incubation of synaptosomes at $\mathrm{pH}_{\mathrm{o}}$ 6.0 for 10 minutes does not change the levels of reduced glutathione. Conversely, treatment with 1-chloro-2,4-dinitrobenzene (CDNB) of the same duration decreased the monochlorobimane fluorescence indicating glutathione depletion (Figure 5). Furthermore, we have shown that the pattern of oxidative stress development detected by DCF upon lowering of $\mathrm{pH}_{\mathrm{o}}$ to 7.0 is similar between control synaptosomes and synaptosomes wherein the pool of reduced glutathione has been depleted by $\mathrm{CDNB}$ (Figure 6).

\section{Discussion}

In order to investigate the process of free radicals accumulation we have used the fluorescent dyes DCFDA and dihydroethidium. DCFDA indicates predominantly levels of highly toxic $\mathrm{OH}$ radicals, while dihydroethidium is able to detect superoxide anion which can function as a signaling molecule apart from its damaging effects. This signaling function may be important for protecting the brain against ischemia (LeBel and Bondy 1990; Halliwell 2006; Ravati et al., 2001; D'Autreaux and Toledano 2007; Niizuma et al. 2009; Kalyanaraman et al. 2012).

We have shown that iron chelator is able to block the $\mathrm{OH}$ radical formation and plasma membrane depolarization, but has no effects on the superoxide anion synthesis and mitochondria depolarization (Figures 1, 2, 3 and 4). Therefore, the presence of free iron is considered to be an essential prerequisite contributing to the damage of presynaptic terminals upon lowering $\mathrm{pH}$. Furthermore, we have shown that the development of significant oxidative stress can potentially be obviated through the use of iron chelators, even when the 

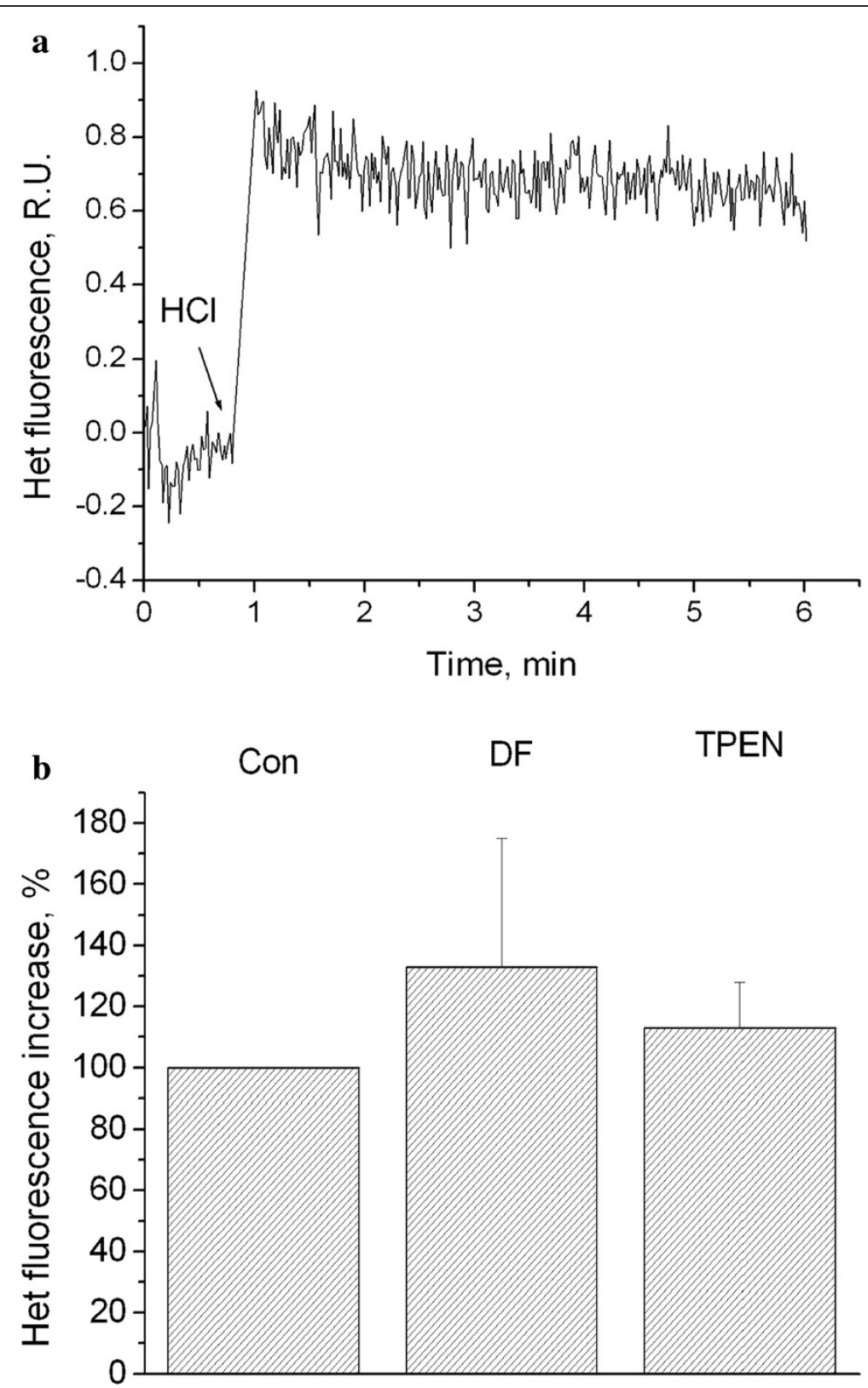

Figure 2 Influence of low pH on dihydroethidium fluorescence in synaptosomes. Role of iron and zinc. a) Kinetics of dihydroethidium fluorescence increase after extracellular acidification. $\mathrm{HCl}$ down to $\mathrm{pH} 6.0$ was added where indicated. Curves represent 22 independent experiments. b) Influence of desferoxamine and TPEN on DCF fluorescence evoked by pH 6.0. Con - control. DF - synaptosomes were preincubated for $30 \mathrm{~min}$ at $37^{\circ} \mathrm{C}$ with $100 \mu \mathrm{M}$ of deferoxamine, incubation medium contains also $100 \mu \mathrm{M}$ of desferoxamine. TPEN - synaptosomes were preincubated for $30 \mathrm{~min}$ at $37^{\circ} \mathrm{C}$ with $5 \mu \mathrm{M}$ of TPEN, incubation medium contains also $5 \mu \mathrm{M}$ of TPEN. Bars represent dihydroethidium fluorescence increase within 4 minutes after additions. Data presented are mean values \pm SEM of at least 4 experiments. 100\% level corresponds to fluorescence increase in response to $\mathrm{pH} 6.0$ without chelators and antioxidants.

superoxide anion synthesis is increased. The presence of iron is also important for the hydroxyl radical formation induced by hydrogen peroxide (Figure 1d). Deferoxamine is able to inhibit oxidative stress induced by both strong and moderate acidification (Figure 1b, c).

The effect of deferoxamine, in terms of smaller oxidative stress induced by hydrogen peroxide, was comparable with that of the classical lipophilic antioxidant ionol (Hocman 1988) (Figure 1d), although the effect was even stronger in the extracellular acidification model (Figure 1b).

Two important conclusions can be drawn based on our experiments aimed at investigating the effect of deferoxamine on plasma membrane and intrasynaptosomal mitochondria potentials. 

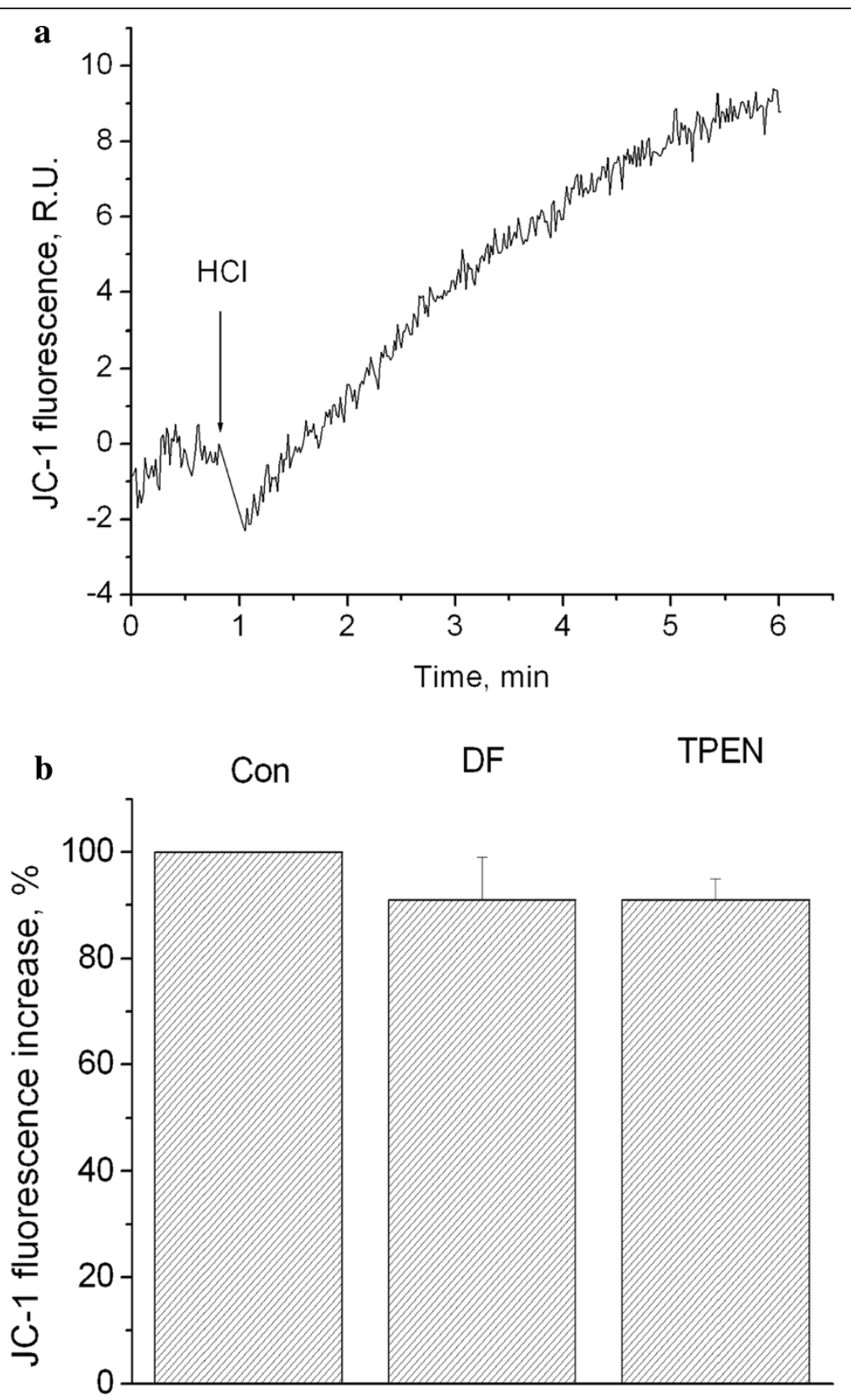

Figure 3 Influence of extracellular pH on JC-1 fluorescence. Role of iron and zinc. a) Kinetics of JC-1 fluorescence increase after extracellular acidification. $\mathrm{HCl}$ down to 6.0 was added where indicated. Curves represent 7 independent experiments. b) Influence of deferoxamine and TPEN on JC-1 fluorescence evoked by $\mathrm{pH}$ 6.0. Con - control. DF - synaptosomes were preincubated for 30 min at $37^{\circ} \mathrm{C}$ with $100 \mu \mathrm{M}$ of deferoxamine, incubation medium contains also $100 \mu \mathrm{M}$ of deferoxamine. TPEN - synaptosomes were preincubated for 30 min at $37^{\circ} \mathrm{C}$ with $5 \mu \mathrm{M}$ of TPEN, incubation medium contains also $5 \mu \mathrm{M}$ of TPEN. Bars represent JC-1 fluorescence increase within 4 minutes after additions. Data presented are mean values \pm SEM of at least 4 experiments. 100\% level corresponds to fluorescence increase in response to pH 6.0.

1) Iron chelating inhibited the plasma membrane depolarization, but not the mitochondria depolarization (Figures 3 and 4). This confirmed our previous findings indicating different mechanisms of acidosis-induced reduction of plasma membrane and mitochondria potentials (Pekun et al. 2014).
2) Our results suggest that ROS are involved in the depolarization of synaptosomal plasma membrane. We have demonstrated previously that the decrease of potential in such case was induced by the inhibition of sodium pump and potassium channels (Fedorovich et al. 2003). Therefore, the free radical-induced damage of sodium 

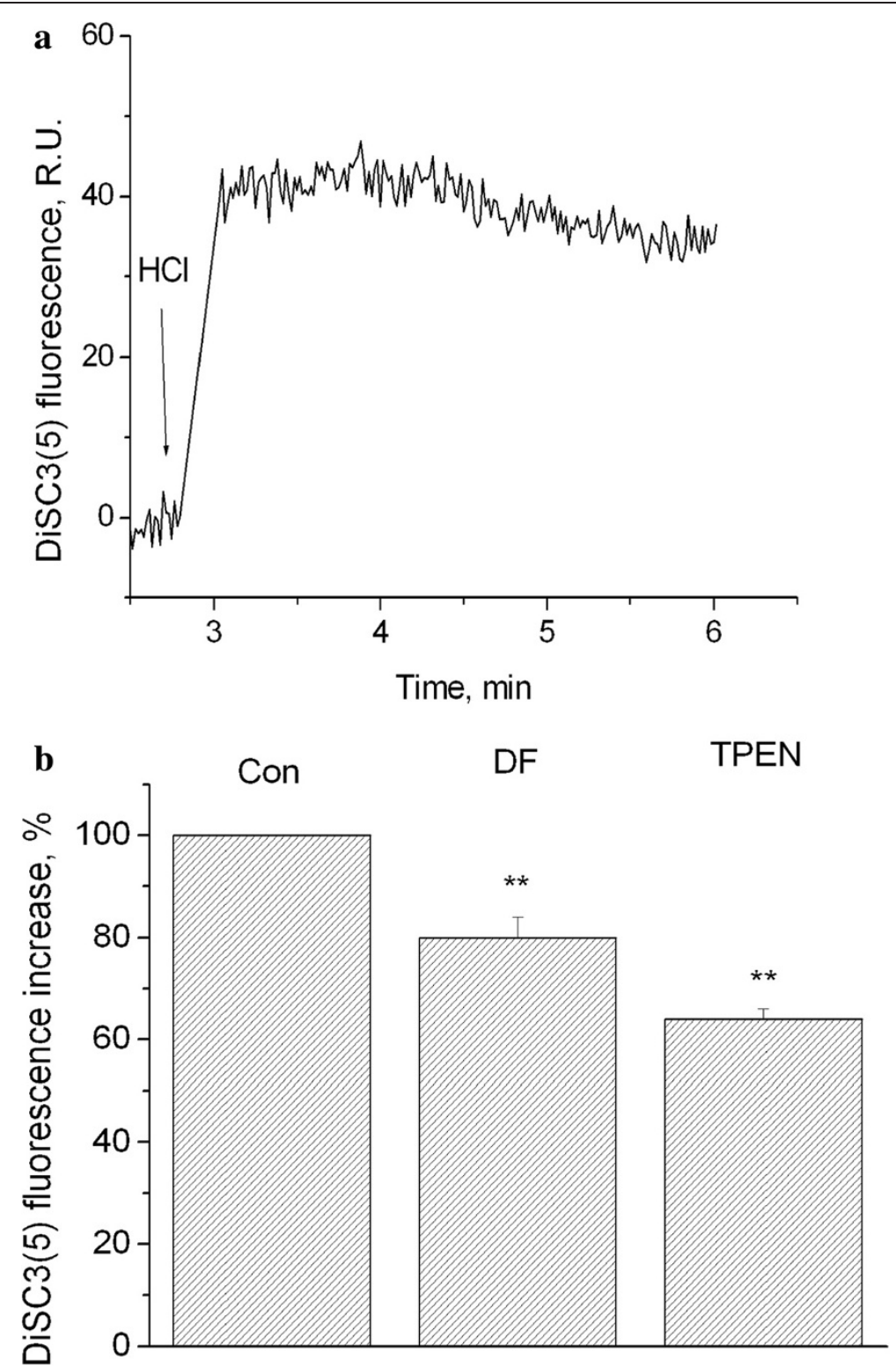

Figure 4 Influence of extracellular pH on DiSC3(5) fluorescence. Role of iron and zinc. a) Kinetics of DiSC3(5) fluorescence increase after extracellular acidification. $\mathrm{HCl}$ down to 6.0 was added where indicated. Curves represents 4 independent experiments. b) Influence of desferoxamine and TPEN on DiSC3(5) fluorescence evoked by pH 6.0. Con - control. DF - synaptosomes were preincubated for $30 \mathrm{~min}$ at $37^{\circ} \mathrm{C}$ with $100 \mu \mathrm{M}$ of deferoxamine, incubation medium contains also $100 \mu \mathrm{M}$ of deferoxamine. TPEN - synaptosomes were preincubated for 30 min at $37^{\circ} \mathrm{C}$ with $5 \mu \mathrm{M}$ of TPEN, incubation medium contains also $5 \mu \mathrm{M}$ of TPEN. Bars represent DiSC3(5) fluorescence increase within 3 minutes after additions. Data presented are mean values \pm SEM of at least 4 experiments. 100\% level corresponds to fluorescence increase in response to $\mathrm{pH}$ 6.0. ${ }^{* *} \mathrm{P} \leq 0.01$ vs. $100 \%$.

pump and/or potassium channels in association with direct influence of protons on potassium channels may underlay the synaptosomal plasma membrane depolarization (Moody 1984).

Our results with chelator TPEN (Figures 2 and 3) rule out the leading role of zinc in mitochondria depolarization and superoxide anion synthesis, as it was shown for other cell models of stroke (Medvedeva et al. 2009; Sensi et al.
2009). However, we show that TPEN is able to inhibit oxidative stress induced by strong but not moderate acidification (Figure 1b, c). Its effect was less pronounced as compared to the effect of deferoxamine (Figure 1b). In this case, the antioxidant properties of TPEN are thought to result from chelating of copper or even chelating of iron with low affinity (Ying et al. 1999; Armstrong et al. 2001; Medvedeva et al. 2009) rather than binding of zinc. 


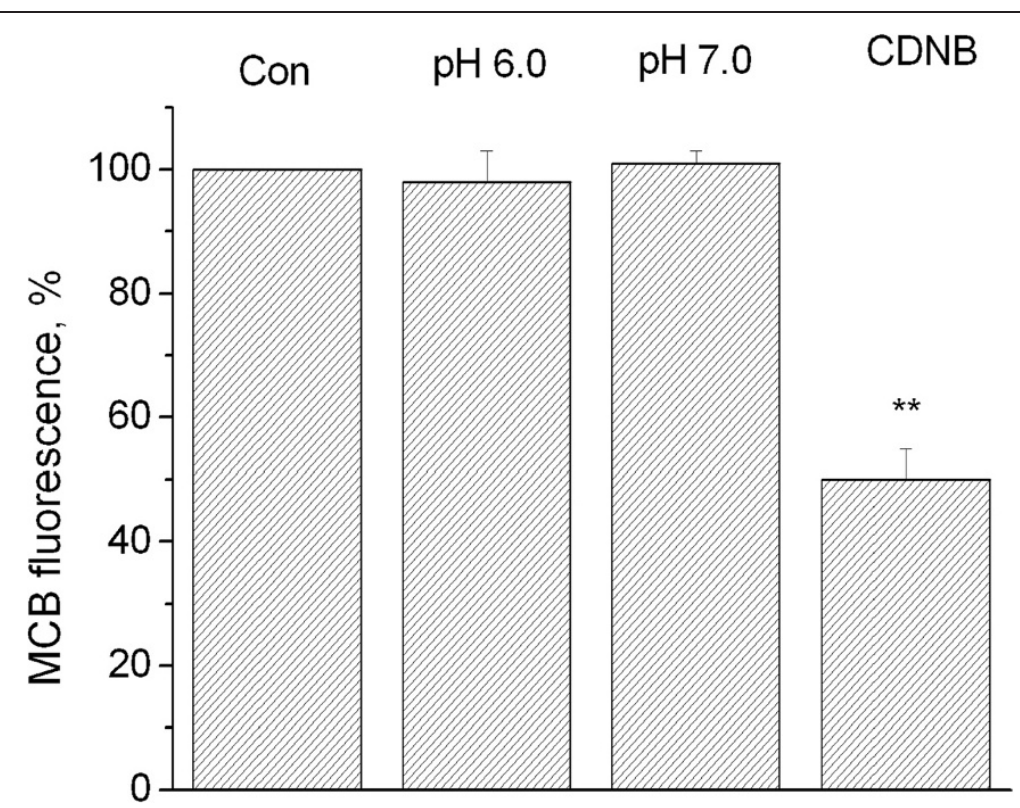

Figure 5 Influence of preincubation at low pH on fluorescence of complex glutathione-monochlorobimane. Con - control. pH $6.0-$ synaptosomes were preincubated for $10 \mathrm{~min}$ at $\mathrm{pH}$ 6.0. $\mathrm{pH} 7.0$ - synaptosomes were preincubated for $10 \mathrm{~min}$ at pH 7.0. CDNB - synaptosomes were preincubated for 10 min with $50 \mu \mathrm{M}$ of CDNB. Data presented are mean values \pm SEM of at least 4 experiments. 100\% level corresponds to fluorescence in control. ${ }^{* *} \mathrm{P} \leq 0.01$ vs. $100 \%$.

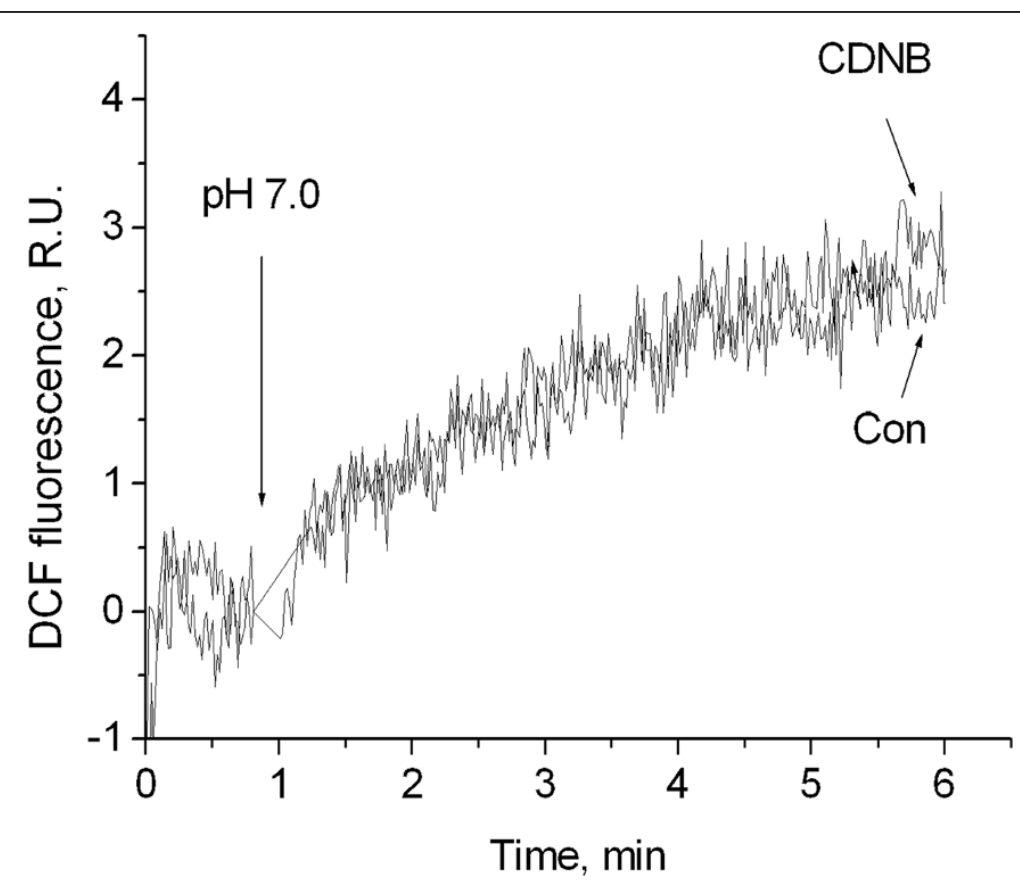

Figure 6 Influence of gluthatione depletion on DCF fluorescence increase evoked by $\mathrm{pH}$ 7.0. $\mathrm{HCl}$ down to $\mathrm{pH} 7.0$ was added where indicated. Con- control. CDNB - synaptosomes were preincubated for 10 min with $50 \mu \mathrm{M}$ of CDNB. 
Although we used a very low TPEN concentration $(5 \mu \mathrm{M})$, we could not exclude a possibility of the involvement of other prooxidant metals.

We have shown that TPEN was able to inhibit the plasma membrane depolarization more strongly than deferoxamine (Figure $4 \mathrm{~b}$ ). This suggests that zinc is more likely involved in the acid-induced decrease of plasma membrane potential than in the decrease of mitochondria potential.

Brain ischemia was shown to change significantly the intracellular levels of reduced glutathione. These levels appeared to be low in ischemic core and surprisingly high in penumbra (Bragin et al. 2010). Furthermore, acidification is found to induce glutathione depletion in neurons (Lewerenz et al. 2010). In contrast, our results clearly show that the levels of reduced glutathione do not change in experimental models used in our studies (Figure 5). In addition, depletion of glutathione by CDNB does not intensify the oxidative stress induced by moderate acidification (Figure 6). Therefore, our results suggest that the reduced glutathione does not contribute significantly to the antioxidant protection of neuronal presynaptic terminals, at least in the acidosis-induced model of oxidative stress.

Deferoxamine exhibited protective effects in some experimental models of brain ischemia in vivo, for instance in ischemia associated with hyperglycemia (Xing et al. 2009) or neonatal brain ischemia (Palmer et al. 1994). It was shown that hyperglycemia could significantly intensify acidification in stroke (Kraig and Chesler 1990; Tombaugh and Sapolsky 1993). Our results provide a possible explanation of deferoxamine efficiency in this case. The clinical trials with administration of deferoxamine for treatment of hemorrhagic stroke have been recently initiated (Xi et al. 2014). Our results indicate that this compound can also be useful in protecting from damage caused by ischemic stroke especially that associated with hyperglycemia. Additionally, the synthetic chelators of iron, VK-28 and HLA-20, which display superior penetrability through blood brain barrier compared to deferoxamine (Zecca et al. 2004) and plant flavonoids, seem to be very promising compounds for the potential treatments of stroke. Flavonoids combine the properties of antioxidants and metal chelators including iron chelators (Afanas'ev et al. 1989; Mandel et al. 2008).

Our results indicate that chelating of iron seems to be a better strategy for the protection of neuronal presynaptic terminals from oxidative stress. This approach obviates the production of highly toxic hydroxyl radicals, but helps to maintain the same level of superoxide anion, which might be important for the protecting brain against ischemia.

\section{Competing interests}

The authors declare that they have no competing interest.

\section{Authors' contribution}

TP and SH performed the experiments; TP, SH and SF analysed the data; TP, TW and SF planned the experiments and wrote the paper. All authors read and approved the final manuscript.

\section{Acknowledgements}

This work was supported by Committee for Aid and Education in Neurochemistry- International Society for Neurochemistry (CAEN-ISN) and Belorussian Republican Foundation of Basic Investigation (grant B13-066). Foundation body had no involvement in study design, in the collection, analysis and interpretation of data, in the writing of the report, and in the decision to submit the article for publication.

\section{Author details}

'Laboratory of Biophysics and Engineering of Cell, Institute of Biophysics and Cell Engineering, Akademicheskaya St., 27, Minsk 220072, Belarus. ${ }^{2}$ Present address: INSERM UMR1106, Institut de Neurosciences des Systems,

Aix-Marseille University, Marseille, France.

Received: 19 September 2014 Accepted: 23 September 2014

Published: 26 September 2014

\section{References}

Abramov AY, Scorziello A, Duchen MR (2007) Three distinct mechanisms generate oxygen free radicals in neurons and contribute to cell death during anoxia and reoxygenation. J Neurosci 27:1129-1138

Afanas'ev IB, Dorozhko Al, Brodskii AV, Kostyuk VA, Potapovich Al (1989) Chelating and free radical scavenging mechanisms of inhibitory action of rutin and quercetin in lipid peroxidation. Biochem Pharmacol 38:1763-1769

Alekseenko AV, Waseem TV, Fedorovich SV (2008) Ferritin, a protein containing iron nanoparticles, induces reactive oxygen species formation and inhibits glutamate uptake in rat brain synaptosomes. Brain Res 1241:193-200

Armstrong C, Leong W, Lees GJ (2001) Comparative effects of metal chelating agents on the neuronal cytotoxicity induced by copper (Cu2+), iron (Fe3+) and zinc in the hippocampus. Brain Res 892:51-62

Bao L, Avshalumov MV, Rice ME (2005) Partial mitochondrial inhibition causes striatal dopamine release suppression and medium spiny neuron depolarization via H2O2 elevation, not ATP depletion. J Neurosci 25:10029-10040

Bragin DE, Zhou B, Ramamoorthy P, Muller WS, Connor JA, Shi H (2010) Differential changes of glutathione levels in astrocytes and neurons in ischemic brains by two-photon imaging. J Cereb Blood Flow Metab 30:734-738

Bralet J, Bouvier C, Schreiber L, Boquilon M (1991) Effect of acidosis on lipid peroxidation in brain slices. Brain Res 539:175-177

Bralet J, Schreiber L, Bouviern C (1992) Effect of acidosis and anoxia on iron delocalization from brain homogenates. Biochem Pharmacol 43:979-983

Chinopoulos C, Tretter L, Adam-Vizi V (1999) Depolarization of in situ mitochondria due to hydrogen peroxide-induced oxidative stress in nerve terminals: inhibition of á-ketoglutarate dehydrogenase. J Neurochem 73:220-228

Crowell JW, Kaufmann BN (1961) Changes in tissue pH after cardiac arrest. Am J Physiol 200:743-745

D'Autreaux B, Toledano MB (2007) ROS as signalling molecules: mechanisms that generate specificity in ROS homeostasis. Nature Rev Mol Cell Biol 8:813-824

Fedorovich SV, Aksentsev SL, Konev SV (1996) Acidosis inhibits calcium accumulation in intrasynaptosomal mitochondria. Acta Neurobiol Exp 56:703

Fedorovich SV, Kaler GV, Konev SV (2003) Effect of low pH on glutamate uptake and release in isolated presynaptic endings from rat brain. Neurochem Res 28:715-721

Giniatullin AR, Darios F, Shakirzyanova A, Davletov B, Giniatullin R (2006) SNAP25 is a pre-synaptic target for the depressant action of reactive oxygen species on transmitter release. J Neurochem 98:1789-1797

Hajos F (1975) An improved method for the preparation of synaptosomal fractions in high purity. Brain Res 93:485-489

Halliwell B (2006) Oxidative stress and neurodegeneration: where are we now? J Neurochem 97:1634-1658

Hocman G (1988) Chemoprevention of cancer: phenolic antioxidants (BHT, BHA). Int J Biochem 20:639-651

Hofmeijer J, van Putten MJAM (2012) Ischemic cerebral damage. An appraisal of synaptic failure. Stroke 43:607-615

Isaev NK, Stelmashook EV, Plotnikov EY, Khryapenkova TG, Lozier ER, Doludin YV Silachev DN, Zorov DB (2008) Role of acidosis, NMDA receptors, and 
acid-sensitive ion channel 1a (ASIC1a) in neuronal death induced by ischemia. Biochemistry (Moscow) 73:1171-1175

Isaev NK, Stelmashook EV, Lukin SV, Freyer D, Mergenthaler P, Zorov DB (2010) Acidosis-induced zinc-dependent death of cultured cerebellar granule neurons. Cell Mol Neurobiol 30:877-883

Kalyanaraman B, Darley-Usmar V, Davies KJA, Dennery PA, Forman HJ, Grisham MB, Mann GE, Moore K, Roberts $L$ 2nd, Ischiropoulos H (2012) Measuring reactive oxygen species and nitrogen species with fluorescent probes: challenges and limitations. Free Rad Biol Med 52:1-6

Kamencic I, Lyon A, Paterson PG, Juurlink BH (2000) Monochlorobimane fluorometric method to measure tissue glutathione. Anal Biochem 286:35-37

Keating DI (2008) Mitochondrial dysfunction, oxidative stress, regulation of exocytosis and their relevance to neurodegenerative diseases. J Neurochem 104:298-305

Kiedrowski L (2011) Cytosolic zinc release and clearance in hippocampal neurons exposed to glutamate - the role of $\mathrm{pH}$ and sodium. J Neurochem 117:231-243

Kraig RP, Chesler M (1990) Astrocytic acidosis in hyperglycemic and complete ischemia. J Cereb Blood Flow Metab 10:104-114

Krishtal OA, Pidoplichko VI (1981) A receptor for protons in the membrane of sensory neurons may participate in nociception. Neuroscience 6:2599-2601

LeBel CP, Bondy SC (1990) Sensitive and rapid quantitation of oxygen reactive species formation in rat synaptosomes. Neurochem Int 17:435-440

Lewerenz J, Dargusch R, Maher P (2010) Lactacidosis modulates glutathione metabolism and oxidative glutamate toxicity. J Neurochem 113:502-514

Lowry O, Rosenbrough H, Farr H, Randall R (1951) Protein measurements with Folin reagent. J Biol Chem 193:265-279

Mandel SA, Amit T, Kalfon L, Reznichenko L, Weinreb O, Youdim MB (2008) Cell signaling pathways and iron chelation in the neurorestorative activity of green tea polyphenols: special reference to epigallocatechin gallate (EGCG). J Alzheimer Dis 15:211-222

Manzanero S, Santro T, Arumugam TV (2013) Neuronal oxidative stress in acute ischemic stroke: sources and contribution to cell injury. Neurochem Int 62:712-718

Medvedeva Y, Lin B, Shuttleworth CW, Weiss JH (2009) Intracellular $\mathrm{Zn}^{2+}$ accumulation contributes to synaptic failure, mitochondrial depolarization, and cell death in an acute slice oxygen-glucose deprivation model of ischemia. J Neurosci 29:1105-1114

Moody W (1984) Effects of intracellular $\mathrm{H}^{+}$on the electrical properties of excitable cells. Annu Rev Neurosci 7:257-278

Nani F, Cifra A, Nistri A (2010) Transient oxidative stress evokes early changes in the functional properties of neonatal rat hypoglossal motoneurons in vitro. Eur J Neurosci 31:951-966

Nedergaard M, Goldman SA, Desai S, Pulsinelli WA (1991) Acid-induced death in neurons and glia. J Neurosci 11:2489-2497

Niizuma K, Endo H, Chan PH (2009) Oxidative stress and mitochondrial dysfunction as determinants of ischemic neuronal death and survival. J Neurochem 109:133-138

Obara M, Szeliga M, Albrecht J (2008) Regulation of pH in the mammalian central nervous system under normal and pathological conditions: fact and hypotheses. Neurochem Int 52:905-919

Palmer C, Roberts RL, Bero C (1994) Deferoxamine posttreatment reduces ischemic brain injury in neonatal rats. Stroke 25:1039-1045

Pekun TG, Waseem TV, Fedorovich SV (2012) Extracellular acidification leads to reactive oxygene species formation in rat brain synaptosomes. Biofizika 57:253-257 (In Russian)

Pekun TG, Lemeshchenko W, Lyskova TI, Waseem TV, Fedorovich SV (2013) Influence of intra- and extracellular acidification on free radical formation and mitochondria membrane potential in rat brain synaptosomes. J Mol Neurosci 49:211-222

Pekun TG, Waseem TV, Fedorovich SV (2014) Depolarization of plasma membrane of rat brain synaptosomes at extra- and intracellular acidification. Biofizika 59:100-104 (In Russian)

Ravati A, Ahlemeyer B, Becker A, Klumpp S, Krieglstein J (2001) Preconditioninginduced neuroprotection is mediated by reactive oxygen species and activation of the transcription factor nuclear factor kappaB. J Neurochem 7:909-919

Sensi SL, Paoletti P, Bush Al, Sekler I (2009) Zinc in the physiology and pathology of the CNS. Nature Rev Neurosci 10:780-791
Siesjo BK, Bendek G, Koide T, Westerberg E, Wieloch T (1985) Influence of acidosis on lipid peroxydation in brain tissues in vitro. J Cereb Blood Flow Metab 5:253-258

Tarasenko A, Krupko O, Himmelreich N (2012) Reactive oxygen species induced by presynaptic glutamate receptor activation is involved in [(3)H]GABA release from rat brain cortical nerve terminals. Neurochem Int 61:1044-1051

Thorn WG, Heitmann R (1954) pH der Gehirnrinde vom Kaninchen in situ während perakuter, totaler Ischämie, reiner Anoxie und in der Erholung. Pflug Arch 218:501-510

Tombaugh GC, Sapolsky RM (1993) Evolving concepts about the role of acidosis in ischemic neuropathology. J Neurochem 61:793-803

Tsentsevitsky A, Kovyazina I, Nikolsky E, Bukharaeva E, Giniatullin R (2013) Redox-sensitive synchronizing action of adenosine on neurotransmitter release at the neuromuscular junction. Neuroscience 248:699-707

Votyakova TV, Reynolds IJ (2001) $\Delta \Psi_{\mathrm{m}}$ - dependent and -independent production of reactive oxygen species by rat brain mitochondria. J Neurochem 79:266-277

Waseem TV, Fedorovich SV (2010) Presynaptic glycine receptors influence plasma membrane potential and glutamate release. Neurochem Res 35:1188-1195

Wemmie JA, Tauhger RJ, Kreple CJ (2013) Acid-sensing ion channels in pain and disease. Nature Rev Neurosci 14:461-471

Xi G, Strahle J, Hua Y, Keep RF (2014) Progress in translation research on intracerebral hemorrhage is there an end in sight? Prog Neurobiol 115:45-63

Xing Y, Hua Y, Keep RF, Xi G (2009) Effects of deferoxamine on brain injury after transient focal cerebral ischemia in rats with hyperglycemia. Brain Res 1291:113-121

Xiong Z-G, Zhu X-M, Chu X-P, Minami M, Hey J, Wei WL, MacDonald JF, Wemmie JA, Price MP, Welsh MJ, Simon RP (2004) Neuroprotection in ischemia: blocking calcium-permeable acid-sensing ion channels. Cell 118:687-698

Yates CM, Butterworth J, Tennant MC, Gordon A (1990) Enzyme activities in relation to $\mathrm{pH}$ and lactate in postmortem brain in Alzheimer-type and other dementia. J Neurochem 55:1624-1630

Ying W, Han S-K, Miller JW, Swanson RA (1999) Acidosis potentiates oxidative neuronal death by multiple mechanisms. J Neurochem 73:1549-1556

Zecca L, Youdim MBH, Riederer P, Connor JR, Crichton RR (2004) Iron, brain ageing and neurodegenerative disorders. Nature Rev Neurosci 5:863-873

doi:10.1186/2193-1801-3-560

Cite this article as: Pekun et al: Role of iron, zinc and reduced glutathione in oxidative stress induction by low $\mathrm{pH}$ in rat brain synaptosomes. SpringerPlus 2014 3:560

\section{Submit your manuscript to a SpringerOpen ${ }^{\odot}$ journal and benefit from:}

- Convenient online submission

- Rigorous peer review

- Immediate publication on acceptance

- Open access: articles freely available online

- High visibility within the field

- Retaining the copyright to your article

Submit your next manuscript at $>$ springeropen.com 